\title{
Ferroelectric film bulk acoustic wave resonators for liquid viscosity sensing
}

\author{
A. Vorobiev ${ }^{\text {a) }}$ and S. Gevorgian \\ Department of Microtechnology and Nanoscience, Chalmers University of Technology, \\ SE-41296 Gothenburg, Sweden
}

(Received 4 June 2013; accepted 11 August 2013; published online 27 August 2013)

\begin{abstract}
A concept of accurate liquid viscosity sensing, using bulk acoustic wave (BAW) resonators, is proposed. The proposed BAW resonators use thin ferroelectric films with the dc field induced piezoelectric effect allowing for generation of pure longitudinal acoustic waves in the thickness excitation mode. This makes it possible to utilize exclusively shear liquid particle displacement at the resonator side walls and, therefore, accurate viscosity evaluation. The BAW resonators with the dc field induced piezoelectric effect in $0.67 \mathrm{BiFeO}_{3}-0.33 \mathrm{BaTiO}_{3}$ ferroelectric films are fabricated and their liquid viscosity sensing properties are characterized. The resonator response is analyzed using simple model of a harmonic oscillator damped by a viscous force. It is shown that the resonator $Q$-factor is inversely proportional to the square root of the viscosity-density product. The viscosity measurement resolution is estimated to be as high as $0.005 \mathrm{mPa} \cdot \mathrm{s}$, which is $0.5 \%$ of the water viscosity. (C) 2013 AIP Publishing LLC. [http://dx.doi.org/10.1063/1.4819327]
\end{abstract}

\section{INTRODUCTION}

Liquid viscosity measurements are used in a wide variety of applications where the liquid flow is a critical part of a product or characterizes the product, such as petroleum, automotive, food, paint, polymer, bio-medical, and many other applications. Development of the high accuracy and precision viscosity sensing is a crucial task. Piezoelectric devices operating in bulk acoustic wave (BAW) mode, such as quartz crystal resonators, have been developed for applications in the advanced viscosity sensing. ${ }^{1,2}$ Their main advantage over conventional viscometers is that they operate without macroscopic moving parts and are therefore more precise and also compact, robust and cheaper to fabricate. Recent improvement of the thin film growth techniques allowed for development of the thin film BAW resonator based liquid sensors. The thin film BAW sensors operate in the same way as the quartz crystal sensors but have the advantage of much higher operation frequency, up to ten gigahertz and above, due to much smaller size. The higher resonance frequency allows for higher sensitivity/resolution since the latter is proportional to frequency. Other advantages of the thin film BAW sensors are CMOS compatibility and much lower manufacturing cost. The thin film BAW sensors are applicable for in-situ and in-vivo viscosity monitoring, which is critical for the most of applications.

It is commonly accepted that the thin film BAW resonators for any practical in-liquid sensing should operate in the shear thickness mode. ${ }^{3,4}$ The reason is that in the conventional BAW sensors the longitudinal thickness mode exhibits particle displacements perpendicular to the resonator surface (out-of-plane) and, thus, generates compressional waves propagating into the liquid, resulting in severe acoustic losses and $Q$-factor degradation. The shear thickness mode displacement is tangential (in-plane), do not emit waves into the liquids and, thus, is able to maintain a high $Q$-factor in

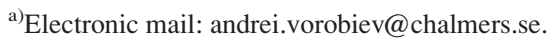

immersed operation. Moreover, specifically tangential displacement of the shear waves couples to the liquid through internal friction forces at the interface and, thus, the resonator response depends on the liquid viscosity. In the reported liquid sensors generation of the shear waves is achieved by using AlN and $\mathrm{ZnO}$ films with tilted polarization vector via fabrication of the films with certain tilt of the crystallographic $c$-axis (Fig. 1(a)). ${ }^{3,4}$ It was shown that in the Newtonian liquids the viscous damping results in the resonator frequency shift proportional to the square root of the viscosity-density product and therefore, knowing the mass density of the liquid, the viscosity can be found. ${ }^{5}$ However, the coupling to the liquid through viscosity only assumes laminar flow at interface. In the devices using tilted polarization this condition is broken. Since the polarization angle is not zero the excited acoustic wave is in a quasi-shear thickness mode. The displacement direction is not perpendicular to the propagation direction, i.e., it is not parallel to the surface, but has a certain deviation angle. ${ }^{6,7}$ In the films with the $c$-axis tilt corresponding to maximum of the coupling coefficient the angle between the wave propagation direction and displacement motion is, approximately, $85^{\circ} .{ }^{7}$ Since liquids exhibit isotropic elastic properties, the quasi-shear thickness mode degenerates at the liquid interface into pure shear and longitudinal components. ${ }^{6,7}$ The longitudinal component emits compressional waves into the liquid, which breaks the condition of the laminar flow at the interface (Fig. 1(a)). According to calculations with the NowotnyBenes model, the dissipation due to the wave emission can be up to $10 \%$ of the total loss balance. ${ }^{4,6}$ This indicates that the disturbance of the laminar flow condition at interface can be significant. Therefore, the coupling to the liquid is not purely viscose. This results in incorrect interpretation of the resonator response and errors in the evaluated liquid parameters.

In this work, we propose a different concept of the BAW resonator liquid viscosity sensing which provides the truly shear particle displacement at a liquid-resonator 

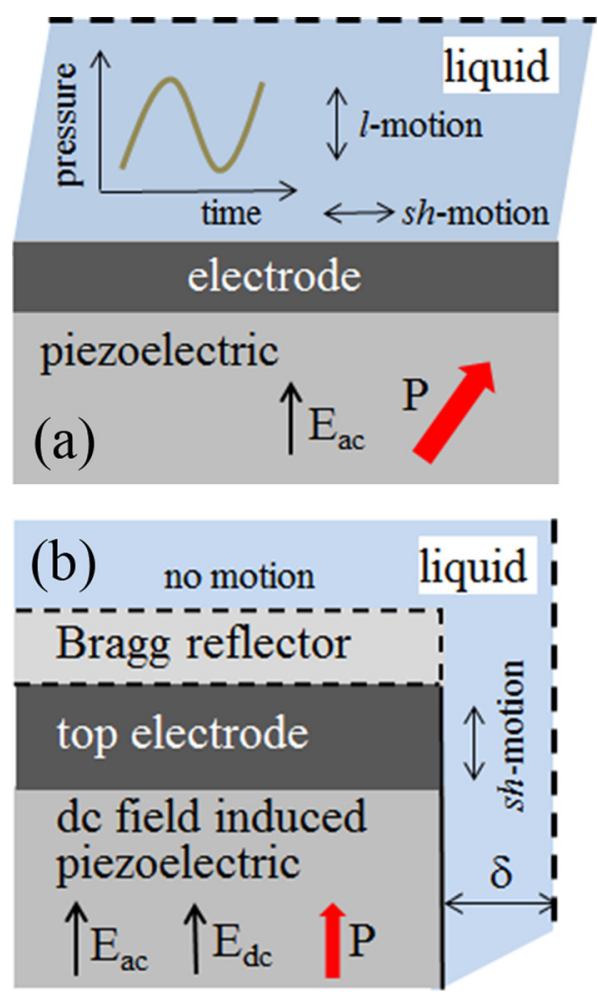

FIG. 1. Schematic diagrams of the resonator-liquid interactions: (a) conventional quasi-shear thickness mode sensor; (b) proposed sensor utilizing the field induced piezoelectric effect (active layer is patterned). The liquid particle motions associated with the longitudinal and shear displacement components are denoted as $l$-motion and sh-motion, respectively.

interface. The proposed resonators utilize ferroelectrics with the dc field induced piezoelectric effect. ${ }^{8}$ In ferroelectrics being in the paraelectric phase the dc field induced polarization follows the applied field direction. In the devices of the parallel-plate configuration, operating in thickness mode, there should not be any angle between the polarization vector and wave propagation direction, as shown in Fig. 1(b). Therefore, excitation of a pure longitudinal thickness mode, with the out-of-plane displacement, is possible. In the case of (101) and (111) crystallographic orientations the shear waves are also exited. However, due to significantly different sound velocities, and, hence, resonant frequencies, the shear amplitudes are negligible. In (001) orientation the shear strain excitation is omitted since the dielectric constant matrix has no off-diagonal elements. The lateral waves associated with $d_{31}$ component of the piezoelectric constant matrix are always excited. However, the lateral sizes of the resonators are too large to have the lateral modes at the same frequency as the thickness mode. Therefore, the effects of the lateral waves can be ignored. In the proposed sensor design, the patterned top electrode/ferroelectric layer stack of the resonator is immersed in the liquid (Fig. 1(b)). The resonator operates in the purely thickness longitudinal mode. This results in exclusively shear particle displacement at the side walls (Fig. 1(b)) and, therefore, only laminar flow of the liquid in this area. A Bragg reflector on the top electrode is proposed with the aim to eliminate leaking of the longitudinal waves into the liquid. Thus, the sensing surface with purely shear displacement is the top electrode/ferroelectric side walls. Analysis indicates that parasitic compressional waves generated at the resonator side walls due to Poisson effect can be ignored. Due to mutual displacement of particles in the areas of highest displacement (i.e., electrode areas) the out-of-plane strain approaches zero. ${ }^{9}$ Therefore, the in-plane strain and displacement are negligible. It should be noted, that the proposed concept of the sensor, where the polarization vector strictly coincides with the liquid surface at the side walls (Fig. 1(b)), can be realized only using mentioned above ferroelectric materials with the dc field induced piezoelectric effect and non-zero polarization component in the dc field direction. The resonators based on other materials, including conventional piezoelectrics ( $\mathrm{AlN}$ or $\mathrm{ZnO})$ or ferroelectrics with spontaneous/remnant polarization (PZT or PMN-PT), operating in the thickness longitudinal mode will always generate the shear wave component, as by product, due to existence of the non-zero components with tilted polarization. If using these materials in the proposed sensors, the shear component of the displacement will generate the compressional waves at the side walls which, again, will disturb the laminar flow conditions.

In this work, we demonstrate the proposed concept of the accurate liquid viscosity sensing. The BAW resonators with the dc field induced piezoelectric effect in ferroelectric films are fabricated and their performance in liquids is investigated experimentally. The resonator response is analyzed using simple model of a harmonic oscillator damped by a viscous force.

\section{EXPERIMENTAL DETAILS}

The test structures are fabricated as BAW solidly mounted resonators on silicon substrates with resistivity of $20 \mathrm{k} \Omega \cdot \mathrm{cm} .{ }^{10}$ The Bragg reflector consisting of two pairs of $\mathrm{SiO}_{2} / \mathrm{W}$ layers with thicknesses of $180 / 180 \mathrm{~nm}$ and $100 / 50 \mathrm{~nm}$ thick $\mathrm{Pt} / \mathrm{TiO}_{2}$ bottom plate are deposited by magnetron sputtering. The $\mathrm{Pt} / \mathrm{TiO}_{2}$ bottom plate is patterned by tilted $\mathrm{Ar}$ ion milling with the aim to provide sloped side walls. The $330 \mathrm{~nm}$ thick ferroelectric films are grown by pulsed laser deposition using a 0.1 wt. $\% \mathrm{MnO}_{2}$ doped $0.67 \mathrm{BiFeO}_{3}-0.33 \mathrm{BaTiO}_{3}(\mathrm{BF}-$ BT) target. ${ }^{11}$ In the resonator design used in these experiments the ferroelectric films are not patterned. The 20/100/ $10 \mathrm{~nm}$ thick $\mathrm{Au} / \mathrm{Al} / \mathrm{Ti}$ top electrode stack is deposited by e-beam evaporation. A lift-off process is used to pattern the top electrodes in the form of circular patches with $44 \mu \mathrm{m}$ in diameter. The top Au film is used for protection of the $\mathrm{Al}$ layer against etching by alkaline developers. The $500 / 50 \mathrm{~nm}$ thick $\mathrm{Au} / \mathrm{Ti}$ stack is deposited by e-beam evaporation and patterned by lift-off process to form leading strips and outer electrodes. The relatively large area of the outer electrodes provides effective dc and ac connection to the Pt bottom plate. ${ }^{10}$ Schematic cross-section view and microphoto of a resonator are shown in Figs. 2 and 3(a), respectively. The microwave response of the resonators is characterized by one port reflection measurements using an Agilent N5230A vector network analyzer and ground-signal-ground (gsg) microprobes in the frequency range 1-10 GHz. Fig. 3(b) shows a microphoto of the measurement set-up. A microtube with $250 \mu \mathrm{m}$ inner diameter is used to deliver the liquids and keep 


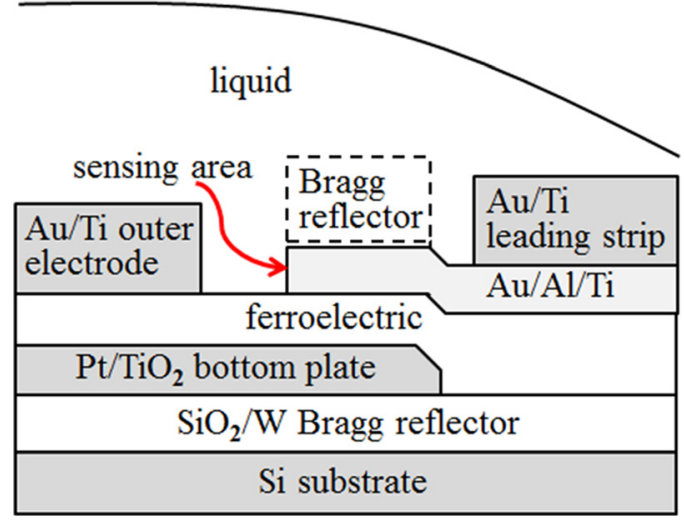

FIG. 2. Schematic cross-section view of the resonator test structure Proposed top Bragg reflector (not used in this work) is also shown.

the liquid layer thickness of about $250 \mu \mathrm{m}$ above the resonator area. Two different liquids have been used in the experiments: Oerlikon Leybold Vacuum paraffin-based mineral oil (N62) and polyalphaolefin oil (SHC224) with physical properties given in Table I. For the sound velocity experimentally measured data (Ref. 12) of the PAO32 and P500N oils, counterparts of the SHC224 and N62, respectively, are used. The oil dynamic viscosity is measured by the falling ball method using RheoTec KF 10 viscometer. The complex input impedance $Z=\operatorname{Re} Z+\mathrm{jIm} Z$ and admittance of the resonators are calculated from the scattering parameters measured in the range $1-10 \mathrm{GHz}$. The series resonance frequency $\left(f_{\mathrm{s}}\right)$ is defined as frequency corresponding to the maximum of the real part of the admittance. Modified Butterworth-Van Dyke circuit
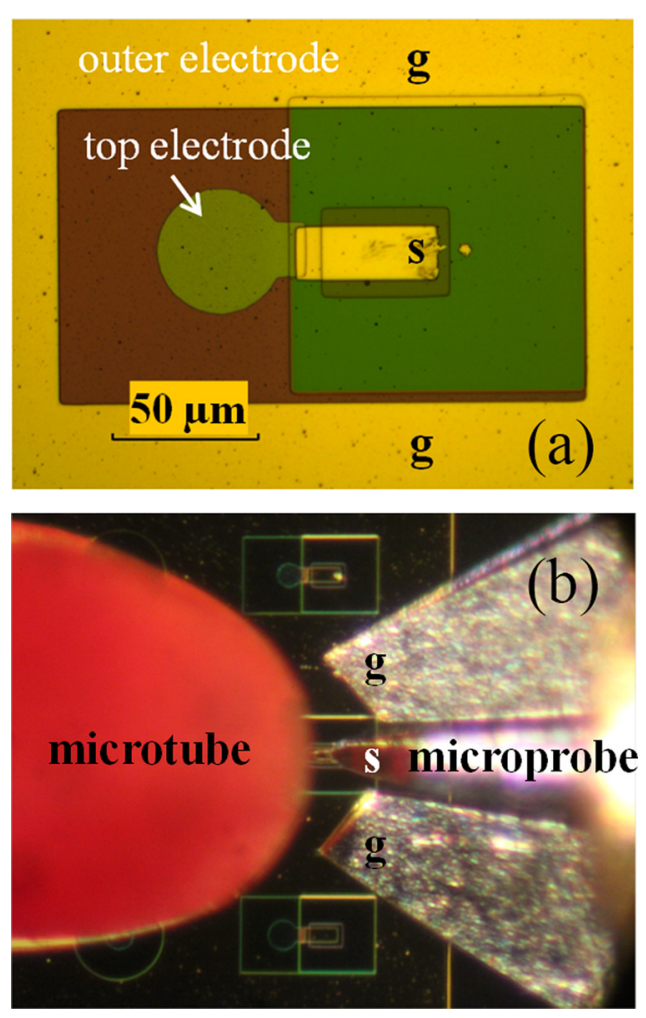

FIG. 3. Microphotos of a resonator test structure (a) and the measurement set-up (b). Contact pads and corresponding signal and ground pins of the microprobe are labeled as "s" and "g," respectively.
TABLE I. Physical properties of oils and the resonator aluminum top electrode at $20^{\circ} \mathrm{C}$.

\begin{tabular}{lccc}
\hline \hline Material & Density $\left(\mathrm{kg} / \mathrm{m}^{3}\right)$ & Sound velocity (m/s) & $\begin{array}{c}\text { Dynamic } \\
\text { viscosity (mPas) }\end{array}$ \\
\hline SHC224 & 830 & 1451 (Ref. 12) & 52 \\
N62 & 880 & 1500 (Ref. 12) & 256 \\
Aluminum & 2700 & 6320 & \\
\hline \hline
\end{tabular}

model is used for de-embedding of the electrical loss associated with series resistance $R_{\mathrm{s}}$ composed mainly by section of the Pt bottom plate between outer and bottom electrodes. ${ }^{13,14}$ The series resistance is found as the real part of impedance at the high frequency limit. ${ }^{14}$ The $Q$-factors are calculated as

$$
Q=\left.\frac{1}{2} f_{\mathrm{s}} \frac{\partial \varphi}{\partial f}\right|_{f=f_{\mathrm{s}}},
$$

where the phase angle is given as $\varphi=\operatorname{arctg}\left[\operatorname{Im} Z /\left(\operatorname{Re} Z-R_{\mathrm{s}}\right)\right]$. The X-ray diffraction (XRD) spectra of the test structures are obtained using a Philips X'pert SW 3040 diffractometer equipped with a point $\mathrm{Cu} \mathrm{K}_{\alpha}$ radiation source, an MRD lens, a thin collimator, and a Ni filter.

\section{RESULTS AND DISCUSSION}

Fig. 4 shows the XRD pattern of a resonator tests structure without the top Au/Ti stack. The peak marked as q(110) is identified as reflection from the strained cristobalite associated with partly crystallized $\mathrm{SiO}_{2}$ layers of the Bragg reflector. It can be seen that there are only reflections from the BF-BT(001) and BF-BT(110) orientations. The BF$\mathrm{BT}(111)$ is probably masked by strong $\mathrm{Pt}(111)$ and $\mathrm{W}(110)$ peaks. Comparison with the XRD pattern of the ceramic counterpart indicates that the films are (001) textured with texturing ratio of, approximately, $3 .^{15,16}$ Structural studies of the ceramic counterparts (for instance, Refs. 15 and 17) did not reveal any deviations from the cubic symmetry. The observed slanted polarization loops and frequency dependent increases in permittivity with temperature can be explained by Maxwell-Wagner-type contribution to the dielectric response, which is very typical for the BFO systems, and not by a ferroelectric-type of polarization. ${ }^{18}$ Additionally, our

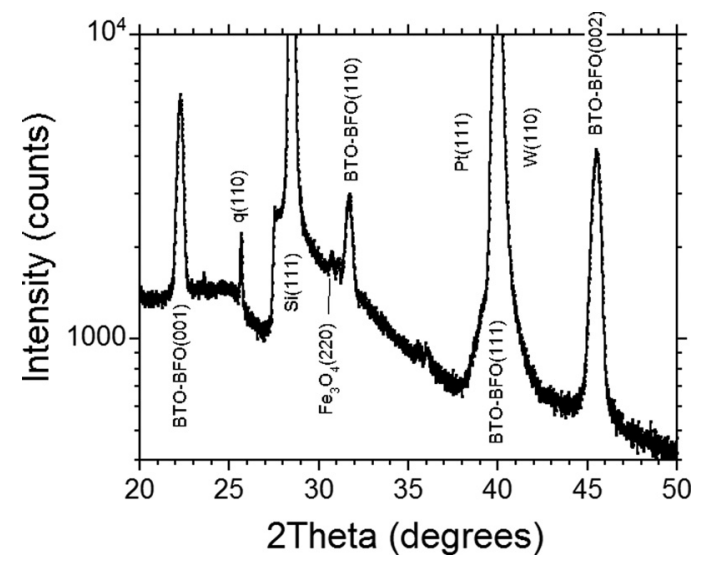

FIG. 4. XRD scan of a resonator tests structure. 
own studies reveal only negligible hysteresis effect in the permittivity at $4.2 \mathrm{GHz}$ (Ref. 11), which also indicates that the material is in paraelectric state, at least at the frequencies of interest.

Fig. 5 shows the normalized real and imaginary parts of admittance of the resonator unloaded and loaded by N62 oil at $10 \mathrm{~V}$ and $9.5 \mathrm{~V}$ dc bias, respectively. Fig. 6 shows the dc bias voltage dependences of the $Q$-factors and the resonance frequencies of the resonators unloaded and loaded by the liquids. The frequencies at $0 \mathrm{~V}$ dc bias are obtained by extrapolating the polynomial fitting curves. The error bars indicate deviations of the results of three independent complete measurement procedures including the liquid delivery. In other cases, the deviations are indicated by the corresponding symbol size. As it can be seen, the $Q$-factor of the resonator loaded by the N62 is lower than that loaded by the SHC224. Below we show that the observed difference in the loaded $Q$-factors is caused by difference in the viscous damping.

We have analyzed the resonator response using simple model of a harmonic oscillator damped by a viscous force. It is assumed that the resonator is subjected to the viscous damping, i.e., the damping force is linearly proportional to the velocity $(u)$ and dynamic viscosity $(\eta)$ in a form of Stokes' law

$$
F_{\mathrm{d}}=\eta A \frac{u}{\delta}=c u,
$$

where $A$ is the area of the moving surface, $\delta$ is the separation between moving boundaries and $c$ is the damping coefficient which is the damping force per unit velocity. In our case, $A$ is the surface area of the top electrode side walls; $\delta$ is the penetration depth of the shear stress (see Fig. 1(b)) ${ }^{19}$

$$
\delta \approx\left(\frac{2 \eta}{\omega_{\mathrm{d}} \rho}\right)^{1 / 2}
$$

where $\omega_{\mathrm{d}}$ is the damped oscillator angular resonance frequency and $\rho$ is the liquid density. The corresponding motion equation of a damped harmonic oscillator is ${ }^{20}$

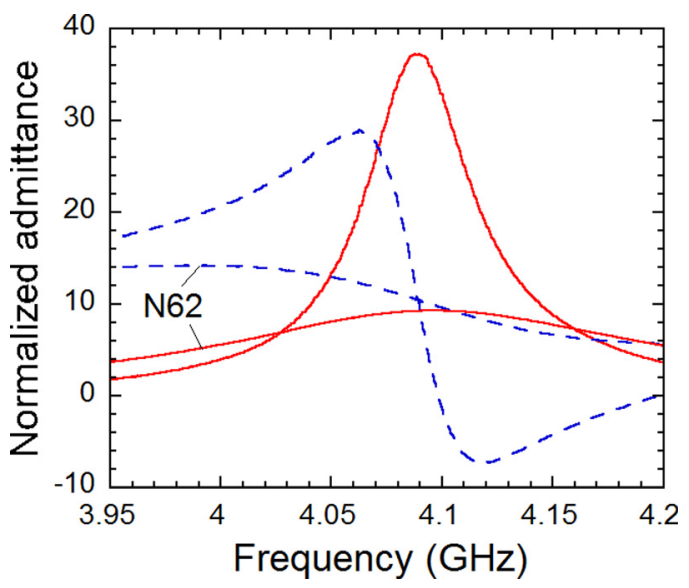

FIG. 5. Normalized real (solid lines) and imaginary (dashed lines) parts of admittance of the resonator unloaded and loaded by N62 oil at $10 \mathrm{~V}$ and $9.5 \mathrm{~V}$ dc bias, respectively.
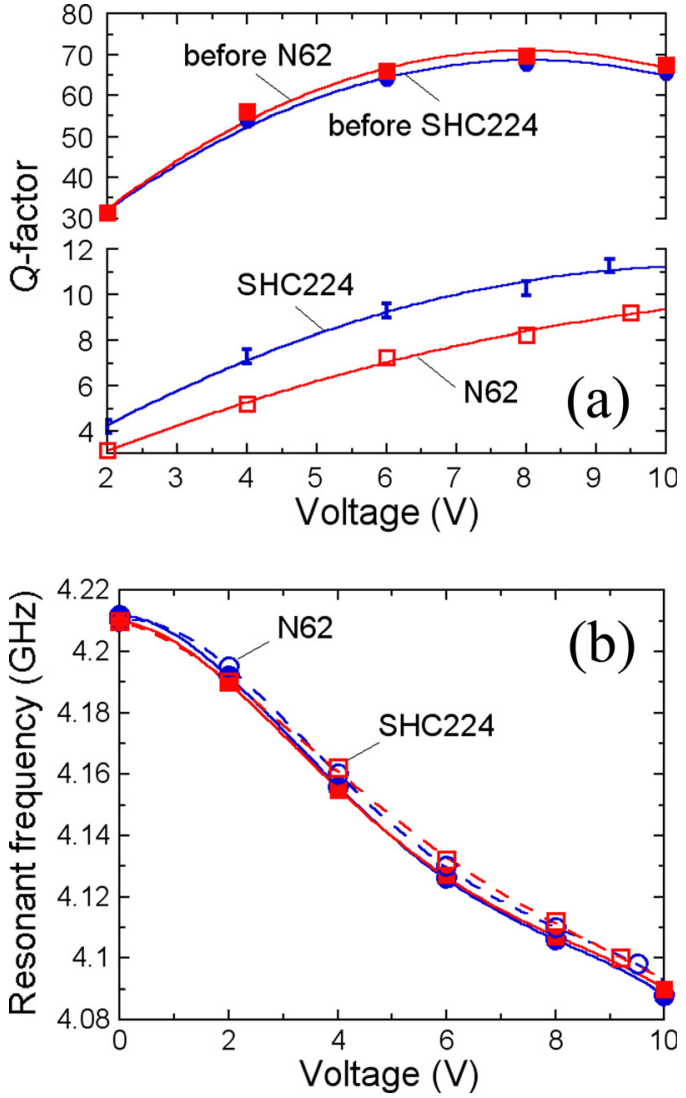

FIG. 6. (a): unloaded $Q$-factors measured directly before applying N62 (solid squares) and SHC224 (solid circles) oils and $Q$-factors of the resonators loaded by N62 (open squares) and SHC224 (error bars) oils vs. dc bias voltage. (b): series resonance frequency of the unloaded resonators (solid symbols, solid lines) and resonators loaded (open symbols, dashed lines) by N62 (circles) and SHC224 (squares) oils vs. dc bias voltage. The lines are polynomial fitting curves.

$$
\ddot{x}+\frac{c}{m} \dot{x}+\omega_{0}^{2} x=0
$$

where $x$ is displacement, $\omega_{0}$ is unloaded resonance frequency, $m$ is the resonator effective mass. The $c / m$ denotes the damping force per unit mass per unit velocity ${ }^{20}$

$$
\frac{c}{m}=2 \xi \omega_{0}
$$

where $\xi$ is the damping ratio (measure of damping). In the case of under-damping $(\xi<1)$, the damped oscillator resonance frequency is ${ }^{20}$

$$
\omega_{\mathrm{d}}=\omega_{0}\left(1-\xi^{2}\right)^{1 / 2}
$$

It can be shown, by using Eqs. (2), (3), and (5), that for the resonators used in our experiments

$$
\xi=\frac{1}{\rho_{s} r}\left(\frac{\eta \rho}{2 \omega_{0}}\right)^{1 / 2},
$$

where $\rho_{\mathrm{s}}$ is the resonator effective mass density and $r$ is the top electrode radius. Estimates show that in our experiments $\xi^{2}$ is below $10^{-6}$, which corresponds to the shift of the resonant frequency $\Delta f=(1 / 2 \pi)\left(\omega_{0}-\omega_{\mathrm{d}}\right)$ less than $3 \mathrm{kHz}$, and is 
much less than the accuracy of the resonance frequency measurements. This explains the observed insensitivity of the resonant frequency to loading by the liquid layers (see Fig. 6(b)).

The $Q$-factor of the loaded resonator can be expressed via $Q$-factors related to the multiple loss mechanisms as

$$
\frac{1}{Q}=\frac{1}{Q_{0}}+\frac{1}{Q_{\text {leak }}}+\frac{1}{Q_{s h}},
$$

where $Q_{0}$ is $Q$-factor of the unloaded resonator, $Q_{\text {leak }}$ is associated with the compressional waves leaking into the liquid and $Q_{\mathrm{sh}}$ is associated with the viscous damping caused by the shear displacement of the liquid at the resonator side walls. In accordance with the basic definition

$$
Q_{\text {leak }}=2 \pi \frac{E_{\text {tot }}}{E_{\mathrm{T}}}=2 \pi \frac{1}{T}=2 \pi \frac{1}{1-R},
$$

where $E_{\text {tot }}$ is the total energy in the system, $E_{\mathrm{T}}$ is energy dissipated per cycle, $T$ and $R$ are the energy transition and reflection coefficients at the resonator-liquid interface, respectively. Since the $20 \mathrm{~nm}$ thick $\mathrm{Au}$ film in the top electrode stack is much thinner than the corresponding wave length its effect can be ignored and the reflection coefficient can be calculated as

$$
R=\left(\frac{\rho_{\mathrm{el}} v_{\mathrm{el}}-\rho_{\mathrm{l}} v_{\mathrm{l}}}{\rho_{\mathrm{el}} v_{\mathrm{el}}+\rho_{\mathrm{l}} v_{\mathrm{l}}}\right)^{2},
$$

where $\rho_{\mathrm{el}}, v_{\mathrm{el}}$ and $\rho_{1}, v_{1}$ are the mass density and sound velocity of the Al top electrode and liquid, respectively. The $Q_{\text {leak }}$ values, obtained using Eqs. (9), (10) and the parameters from Table I, are 25.6 and 23.8 for the SHC224 and N62 oils, respectively. Then, the de-embedded $Q$-factors are calculated using Eq. (8) as

$$
Q_{\mathrm{de}}=\left(\frac{1}{Q}-\frac{1}{Q_{\text {leak }}}\right)^{-1}
$$

and are 20.2 and 15.0 for the SHC224 and N62 oils, respectively, at $10 \mathrm{~V}$ dc bias. In general, the de-embedding procedure mimics effect of the proposed top Bragg reflector, although the $Q$-factors will be different due to contribution of the real Bragg reflector to the resonator effective mass. Equation (8) can be rewritten as

$$
Q_{\mathrm{de}}=\left(\frac{1}{Q_{0}}+\frac{1}{Q_{\mathrm{sh}}}\right)^{-1} .
$$

The viscously damped oscillator $Q$-factor is ${ }^{20}$

$$
Q_{\mathrm{sh}}=\frac{1}{2 \xi} \text {. }
$$

By using Eqs. (5), (2), and (3), Eq. (13) can be rewritten as

$$
Q_{\mathrm{sh}}=\frac{m}{A}\left(\frac{2 \omega_{\mathrm{d}}}{\eta \rho}\right)^{1 / 2} .
$$

It can be seen that the $Q$-factor associated with the viscous damping is inversely proportional to the square root of the viscosity-density product and therefore, knowing the mass density of the liquid, the viscosity can be determined. Fig. 7(a) shows the de-embedded $Q$-factors of the resonators (solid circles) versus the liquid viscosity-density product at $10 \mathrm{~V} \mathrm{dc}$ bias. The solid line is the best-fit curve obtained using Eqs. (12) and (14) for the resonator effective mass $m=1.4 \cdot 10^{-14} \mathrm{~kg}$. The model is valid in the case of underdamped system, i.e., when $Q>1 / 2 .^{20}$ Then, by using Eq. (14), it may be shown that, for example, the resonator loaded by the N62 oil will be underdamped if the effective mass is more than $4.5 \cdot 10^{-16} \mathrm{~kg}$. However, as it will be shown below the effective mass is only a small part of the top electrode plate mass. It can be seen that the experimental points reveal slightly weaker dependence on the viscositydensity product than that predicted by the model. The model assumes that the velocity of the moving surface is small enough to drag the adjacent liquid layer with the same velocity. This condition is, most likely, broken at the rather high resonant frequency of ca. $4 \mathrm{GHz}$ used in the experiments, which may explain the observed weaker $Q$-factor dependence. However, this is a feature of the particular experiments but not an inherent drawback of the sensor concept. The resonant frequency can be significantly reduced by increasing thickness of the ferroelectric film and/or electrodes.

Fig. 7(a) shows also simulated $Q$-factor (dashed line) of a resonator having the same mass and dimensions but higher unloaded $Q_{0}=360$, which is measured using the circular resonator test structures with $\mathrm{Ba}_{0.25} \mathrm{Sr}_{0.75} \mathrm{TiO}_{3}$ films grown at optimized conditions. ${ }^{10}$

The added mass of the $250 \mathrm{~nm}$ thick liquid layers, used in the experiments, is approximately $2 \cdot 10^{-10} \mathrm{~kg}$, which is
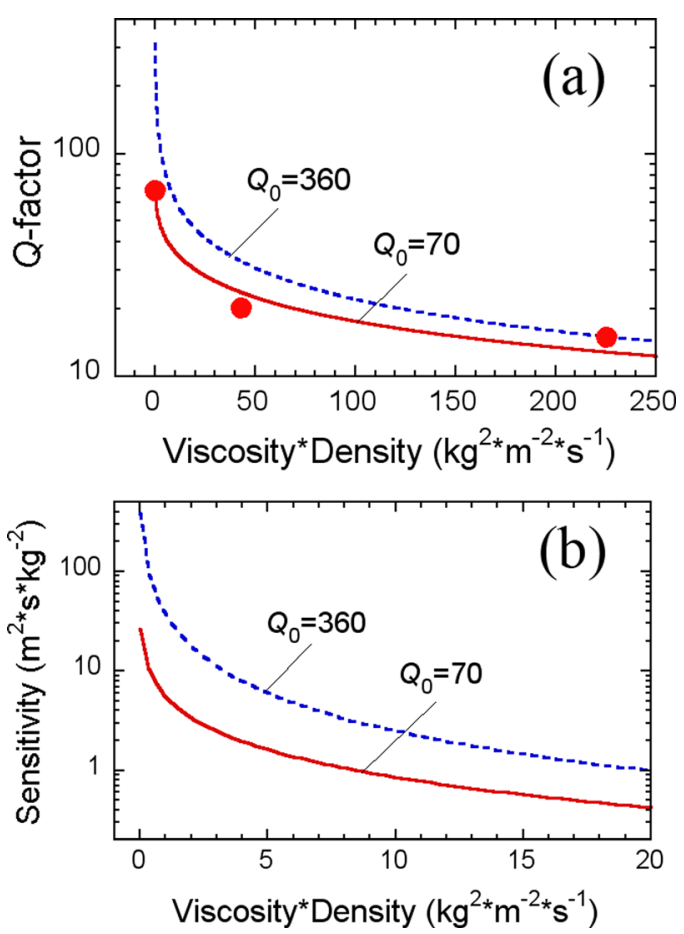

FIG. 7. $Q$-factors (a) and sensitivity (b) of the resonators at $10 \mathrm{~V}$ dc bias vs. viscosity-density product. The $Q$-factor lines are fitting curves. 
much larger than the resonator effective mass. This confirms condition of the total immersion of the resonator surfaces in the viscous liquid. The coupling to the liquid is through viscosity and leaking compressional waves only. There are no any gravimetric effects.

The viscosity sensitivity of the $Q$-factor can be defined as in Ref. 21

$$
S=-\frac{\partial Q}{\partial(\eta \rho)} .
$$

Fig. 7(b) shows sensitivity calculated as derivative of the $Q$-factor versus viscosity-density product fitting curves for both unloaded $Q$-factors $Q_{0}=70$ and $Q_{0}=360$. It can be seen that the sensitivity is strongly dependent on the viscosity-density product and limited by the unloaded $Q$-factor. The sensitivity dependences allows for estimation of the viscosity measurement resolution. For example, it can be seen from Fig. 7(b) that the sensitivity of the resonator with $Q_{0}=360$ loaded by water (the viscosity-density product is ca. $\left.1 \mathrm{~kg}^{2} \cdot \mathrm{m}^{-2} \cdot \mathrm{s}^{-1}\right)$ is ca. $50 \mathrm{~m}^{2} \cdot \mathrm{s} \cdot \mathrm{kg}^{-2}$. With assumed $Q$-factor resolution in water of 0.25 (Ref. 21) the viscosity resolution will be $0.005 \mathrm{mPa} \cdot \mathrm{s}$, which is $0.5 \%$ of the water viscosity. This is already high enough for the accurate characterization of water and other low viscosity liquids. On the other hand, the viscosity of majority of liquids, oils for example, can be effectively reduced down to $10 \mathrm{mPa} \cdot \mathrm{s}$ and less by a soft heating to the temperatures less than $100^{\circ} \mathrm{C} .{ }^{12}$ This leads to an additional advantage of the ferroelectric based sensors which is possibility of simultaneous monitoring and/or controlling (by heating/cooling) of temperature of the liquid under test using the inherent temperature dependence of the ferroelectric permittivity. ${ }^{22}$ This possibility may allow for developing of multifunctional integrated sensor systems. By using Eq. (14) and assuming that the viscous damping dominates in the total loss balance the sensitivity can be derived to

$$
S=\frac{m}{2 A}\left(\frac{2 \omega_{\mathrm{d}}}{(\eta \rho)^{3}}\right)^{1 / 2} .
$$

Thus, the sensitivity is proportional to the resonant frequency and to the inverse sensing area. Therefore, the sensitivity can be increased by decreasing the top electrode thickness due to both increase in the resonant frequency and decrease in the sensing area. The inverse dependence on the sensing area follows from the fact that sensitivity is proportional to the $Q$ factor, which is proportional to the inverse sensing area. It should be noted here that the resonator effective mass $\left(m=1.4 \cdot 10^{-14} \mathrm{~kg}\right)$ is much less than the corresponding mass of the top electrode $\left(m=4.1 \cdot 10^{-13} \mathrm{~kg}\right)$. This indicates that the top electrode contributes mainly to the elastic properties of the resonator. Therefore one can expect that decrease in the sensing area with the top electrode thickness will be not fully compensated by corresponding decrease in the resonator effective mass. Approximation (16) is valid where the viscous loss dominates in the total loss balance, see Eq. (12). With significant reduction of the top electrode thickness, down to the values where contribution of the viscous loss is minor, sensitivity decreases. This effect may appear at higher resonance frequencies where the thinner electrodes are required. However, it can be always compensated by increase in the top electrode radius giving rise to the sensing area.

\section{SUMMARY}

A concept of accurate liquid viscosity sensing using BAW resonators is demonstrated. The proposed resonators utilize thin films of ferroelectric materials which reveal dc field induced piezoelectric effect. In ferroelectrics being in the paraelectric phase, the dc field induced polarization follows the applied field direction. In the devices of the parallel-plate configuration, operating in thickness mode, the angle between the polarization vector and wave propagation direction is zero. At these conditions a pure longitudinal thickness mode can be generated. This allows for realization of exclusively shear particle displacement at the resonator side walls and, thus, only laminar flow of the liquid. Therefore, the liquid viscosity can be measured accurately. In the proposed sensor concept a Bragg reflector on the top electrode is used with the aim to eliminate leaking of the longitudinal waves into the liquid.

The dc field induced $\mathrm{BAW}$ resonators based on the $0.67 \mathrm{BiFeO}_{3}-0.33 \mathrm{BaTiO}_{3}$ ferroelectric are fabricated. The XRD analysis reveals (001) texture with texturing ratio of, approximately, 3 . The $Q$-factor and resonance frequency of the resonators are studied as response to loading by the liquids with different dynamic viscosities up to $256 \mathrm{mPa} \cdot \mathrm{s}$. The resonator response is analyzed using simple model of a harmonic oscillator damped by a viscous force. It was found that due to low damping ratio (less than $10^{-3}$ ) the shift of the loaded resonant frequency is less than accuracy of measurements. On the other hand, the loaded resonator $Q$-factor reveals a dependence on the liquid viscosity and can be reproducibly measured. The model predicts that the resonator $Q$-factor is inversely proportional to the square root of the viscosity-density product. The experimental points reveal slightly weaker dependence which is explained by rather high resonant frequency used in the experiments. The $Q$-factor viscosity sensitivity depends on the viscosity-density product and limited by the unloaded $Q$-factor. The viscosity measurement resolution is estimated to be as high as $0.005 \mathrm{mPa} \cdot \mathrm{s}$, which is $0.5 \%$ of the water viscosity. The inherent temperature dependence of the ferroelectric permittivity allows for monitoring and/or controlling of temperature of the liquid under test. This makes it possible to develop multifunctional integrated sensor systems.

\section{ACKNOWLEDGMENTS}

This work was supported by the project NAFERBio, Vinnova, Sweden.

${ }^{1}$ A. Agoston, C. Ötsch, and B. Jakoby, Sens. Actuators, A 121, 327 (2005).

${ }^{2}$ D. Wang, P. Mousavi, P. J. Hauser, W. Oxenham, and C. S. Granta, Colloids Surf., A 268, 30 (2005).

${ }^{3}$ E. Milyutin and P. Muralt, "Thin film bulk acoustic wave resonators for gravimetric sensing," in Nanosystems Design and Technology, edited by G. DeMicheli, Y. Leblebici, M. Gijs, and J. Vörös (Springer Science, London, 2009), p. 103. 
${ }^{4}$ G. Wingqvist, J. Bjurström, L. Liljeholm, V. Yantchev, and I. Katardjiev, Sens. Actuators B 123, 466 (2007).

${ }^{5}$ K. K. Kanazawa and J. G. Gordon, Anal. Chem. 57, 1770 (1985).

${ }^{6}$ G. Wingqvist, PhD. thesis, Uppsala University, Sweden, 2009.

${ }^{7}$ T. Yanagitani, "Shear mode piezoelectric thin film resonators," in Acoustic Waves-From Microdevices to Helioseismology, edited by M. G. Beghi (InTech, 2011), pp. 506-507.

${ }^{8}$ S. Gevorgian, A. Tagantsev, and A. Vorobiev, Tuneable Bulk Acoustic Wave Resonators (Springer, London, 2013).

${ }^{9} \mathrm{~K} .-\mathrm{y}$. Hashimoto, RF Bulk Acoustic Wave Filters for Communications (Artech House, Norwood, MA, 2009), p. 75.

${ }^{10}$ A. Vorobiev and S. Gevorgian, Appl. Phys. Lett. 96, 212904 (2010).

${ }^{11}$ A. Vorobiev, S. Gevorgian, N. Martirosyan, M. Löffler, and E. Olsson, Appl. Phys. Lett. 101, 232903 (2012).

${ }^{12}$ S. Mia, Ph.D. thesis, Saga University, Japan, 2010.

${ }^{13}$ M. Norling, J. Berge, and S. Gevorgian, IEEE MTT-S Int. Microwave Symp. Dig. 1, 101 (2009)
${ }^{14}$ A. A. Vorobiev, S. Gevorgian, M. Löffler, and E. Olsson, J. Appl. Phys. 110, 054102 (2011).

${ }^{15}$ S. O. Leontsev and R. E. Eitel, J. Am. Ceram. Soc. 92, 2957 (2009).

${ }^{16}$ P. Padmini, T. R. Taylor, M. J. Lefevre, A. S. Nagra, R. A. York, and J. S. Speck, Appl. Phys. Lett. 75, 3186 (1999).

${ }^{17}$ M. M. Kumar, A. Srinivas, and S. V. Suryanarayana, J. Appl. Phys. 87, 855 (2000).

${ }^{18}$ S. Kamba, D. Nuzhnyy, M. Savinov, J. Šebek, J. Petzelt, J. Prokleška, R. Haumont, and J. Kreisel, Phys. Rev. B 75, 024403 (2007).

${ }^{19}$ D. S. Ballantine, R. M. White, S. J. Martin, A. J. Ricco, E. T. Zellers, G. C. Frye, and H. Wohltjen, Acoustic Wave Sensors; Theory, Design, and Physico-Chemical Applications (Academic Press, 1997).

${ }^{20}$ M. R. Srinivasan, Physics for Engineers (New Age International Pvt Ltd Publishers, 2009).

${ }^{21}$ J. Weber, M. Link, R. Primig, D. Pitzer, and M. Schreiter, International Frequency Control Sym. Dig. 1, 117 (2006).

${ }^{22}$ A. Vorobiev and S. Gevorgian, J. Eur. Ceram. Soc. 27, 3847 (2007). 